\title{
Two-phonon process and hyperfine interaction limiting slow hole-spin relaxation time in InAs/GaAs quantum dots
}

\author{
F. Fras, B. Eble, P. Desfonds, F. Bernardot, C. Testelin, and M. Chamarro* \\ Institut des NanoSciences de Paris, UPMC Univ Paris 06, CNRS UMR 7588, 4 Place Jussieu, F-75252 Paris Cedex 05, France
}

A. Miard and A. Lemaître

Laboratoire de Photonique et Nanostructures, CNRS, Route de Nozay, F-91460 Marcoussis, France

(Received 23 February 2012; published 9 July 2012)

\begin{abstract}
We study the hole-spin relaxation in p-doped InAs quantum dots. Two relaxation mechanisms are evidenced, at low magnetic field $(0 \leqslant B \leqslant 2 T)$ and low temperature $(2 \leqslant T \leqslant 50 K)$, by using a pump-probe configuration and a recent experimental technique working in the frequency domain. At $T=2 K$, the coupling to nuclear spins and the hole wave-function inhomogeneity fix the hole-spin relaxation rate value, $\Gamma_{1}^{h} \approx 1 \mu \mathrm{s}^{-1}$. It decreases with increasing magnetic field and reaches a plateau at $0.4 \mu \mathrm{s}^{-1}$. At $T \geqslant 7 \mathrm{~K}$, two-phonon spin-orbit process dominates and leads to a quadratic temperature dependence of $\Gamma_{1}^{h}$, in good agreement with theory.
\end{abstract}

DOI: 10.1103/PhysRevB.86.045306

PACS number(s): 72.25.Rb, 72.25.Fe, 78.55.Cr, 78.67.Hc

\section{INTRODUCTION}

A single spin confined in a quantum dot (QD) is a potential candidate for applications in nanoscale spintronics and as a solid-state qubit. ${ }^{1,2}$ Until recently, most studies have used the spin of an electron as a support of information; however, a major difficulty of using electron spins is the strong hyperfine (hf) interaction which couples the electronic spin to roughly $N \sim 10^{5}$ nuclei. The electronic spin undergoes, at low temperature and in absence of an external magnetic field, an effective fluctuating magnetic field on the order of tens of $\mathrm{mT}$, which fixes its dephasing time in the order of ns. ${ }^{3,4}$ Isotopic engineering may reduce nuclear noise, but there is no stable spin-zero isotope for III-V semiconductor compounds. That is why a single hole spin confined in a QD has emerged as an alternative candidate, having an order-of-magnitude weaker hf interaction. ${ }^{5-8}$ Moreover, for a heavy hole $(\mathrm{HH})$, the hf interaction has an Ising-like form, in contrast to the Heisenberg-type interaction of electrons. ${ }^{9,10}$ The absence of transverse hf terms has important consequences for the hole-spin dynamics. In particular, no dephasing can be observed for an $\mathrm{HH}$ spin initialized along the growth direction [Fig. 1 in Ref. 10]. However, because hole states show high sensitivity to the anisotropy of local electrostatic fields, strains, and variations of the confinement potentials, signatures of the $\mathrm{HH}$ and light-hole (LH) mixing have experimentally been observed in real QDs. ${ }^{10,11}$ The hole mixing is at the origin of a transverse hole hf-interaction term, and recently, a hole-spin dephasing time, associated with the carrier spin precession in a random frozen nuclear field and in the order of $10 \mathrm{~ns}$, has been reported in InAs QDs. ${ }^{5}$ Theoretical works also predict that the $\mathrm{hf}$ interaction can be at the origin of another slower carrier-spin relaxation time, falling in the microsecond range and related to the nuclear spin-bath dynamics. ${ }^{3,4,12-17}$ In this way, at low temperature and in absence of an applied magnetic field, Pal et al. ${ }^{18}$ have measured an electron-spin relaxation time equal to $1 \mu \mathrm{s}$. Moreover, when a magnetic field is applied, the presence of a Zeeman splitting introduces new channels of relaxation via phonon-assisted spin flips mediated by spin-orbit. In moderate magnetic fields (2-10 T) and at low temperature, recent experimental works show that the electron
$T_{1}^{e}$ and the hole $T_{1}^{h}$ spin relaxation times, in the range of milliseconds, are governed by the same mechanism, i.e. the spin-orbit-mediated single-phonon scattering. ${ }^{19,20}$ However, at low magnetic field, as the Zeeman splitting is very small, the density of phonons able to match the Zeeman energy vanishes, and then this mechanism becomes negligible. ${ }^{21-23}$ Hyperfinephonon-mediated mechanisms have been also proposed, but the spin relaxation rate is zero in absence of magnetic field as for one-phonon-assisted process. ${ }^{21}$

In this paper, we address the slow hole-spin relaxation mechanisms in conditions of low magnetic field $(0 \leqslant B \leqslant$ $2 \mathrm{~T})$ and low temperature $(2 \leqslant T \leqslant 50 \mathrm{~K})$, which have been largely unexplored. ${ }^{24} \mathrm{~A}$ recently proposed experimental method, ${ }^{25}$ dark-bright time-scanning spectroscopy (DTS), based on differential absorption measurements and working in the frequency domain, is used to measure $T_{1}^{h}$ as a function of a longitudinal magnetic field and of lattice temperature. We experimentally show that the slow $T_{1}^{h}$ is limited by two mechanisms in InAs QDs: at $T=2 \mathrm{~K}$, the hf coupling with the nuclear spins of the host material fixes the $T_{1}^{h}$ value at $\approx 1 \mu \mathrm{s}$ and imposes an increase for very low magnetic fields in the range of tens of $\mathrm{mT}$; for larger fields, $T_{1}^{h}$ saturates at $\approx 2.5 \mu$ s. Additionally, our temperature study constitutes experimental evidence of the importance of the two-phononassisted mechanism (TPAM) on the hole-spin relaxation, and it leads to a quadratic dependence for the hole-spin relaxation rate $\Gamma_{1}^{h}=1 / T_{1}^{h}$ with temperature in the $7-50 \mathrm{~K}$ range, in good agreement with theoretical predictions. ${ }^{21,26}$

\section{SAMPLE AND EXPERIMENTAL SETUP}

Quantum dots were grown by molecular-beam epitaxy on a (001) GaAs substrate. The QD surface density is about $10^{10} \mathrm{~cm}^{-2}$. The studied samples were p-modulation doped with a Carbon $\delta$-doping layer located below each of their 30 QD layers at $2 \mathrm{~nm}$ with a nominal density of $\sim 1.2 \times$ $10^{11} \mathrm{~cm}^{-2}$ (Sample A) or $2 \times 10^{11} \mathrm{~cm}^{-2}$ (Sample B). The sample is placed either in a helium-bath cryostat containing superconducting coils or in a zero-field He-continuous-flow cryostat for temperature measurements from 4 to $50 \mathrm{~K}$. In 
order to improve the QD temperature monitoring in this latter cryostat, a cold thermic screen with a small optical access was used, and a calibrated temperature sensor was inserted in the sample vicinity.

A permanent train of 2-ps pulses from a mode-locked Ti:sapphire laser with a 76- $\mathrm{MHz}$ repetition rate is split into pump and probe beams. The pump-beam polarization is circular. The probe beam is linearly polarized; after transmission through the sample, the probe beam is decomposed into its two circular components. The difference of their intensities is then measured with a balanced optical bridge to obtain the signal of photo-induced circular dichroism (PCD) in the studied QD samples.

\section{EXPERIMENTAL RESULTS AND DISCUSSION}

\section{A. Magnetic field dependence of the hole-spin relaxation rate}

The spin initialization of hole spins can be obtained by an optical pulsed excitation, circularly polarized and resonant with the trion transition. ${ }^{25,27}$ Figure 1 shows the temporal behavior of the PCD measured when pump and probe beams are tuned at $1.32 \mathrm{eV}$, the lowest optical transition in p-doped QDs. The PCD signal at negative pump-probe delays indicates that the spin polarization is not fully relaxed within the $T_{L}=13 \mathrm{~ns}$ repetition period of the laser pulses and has been unambiguously associated to the net spin polarization of the resident holes. In DTS experiments, a pump-probe configuration is used to separate the long-living hole-spin polarization from the short-living photo-created trion-spin one. The pump beam intensity is switched on and off periodically by an acousto-optic modulator (AOM; see inset in the upper part of Fig. 1), producing bright and dark excitation intervals of variable duration, with rise and fall times $\leqslant 50 \mathrm{~ns}$. In this way, the pump excitation is a periodic function, as well as the measured PCD $\left(T_{L}\right)$ signal (see inset in the lower part of Fig. 1). We measure the second Fourier coefficient of $\operatorname{PCD}\left(T_{L}\right)$ as a

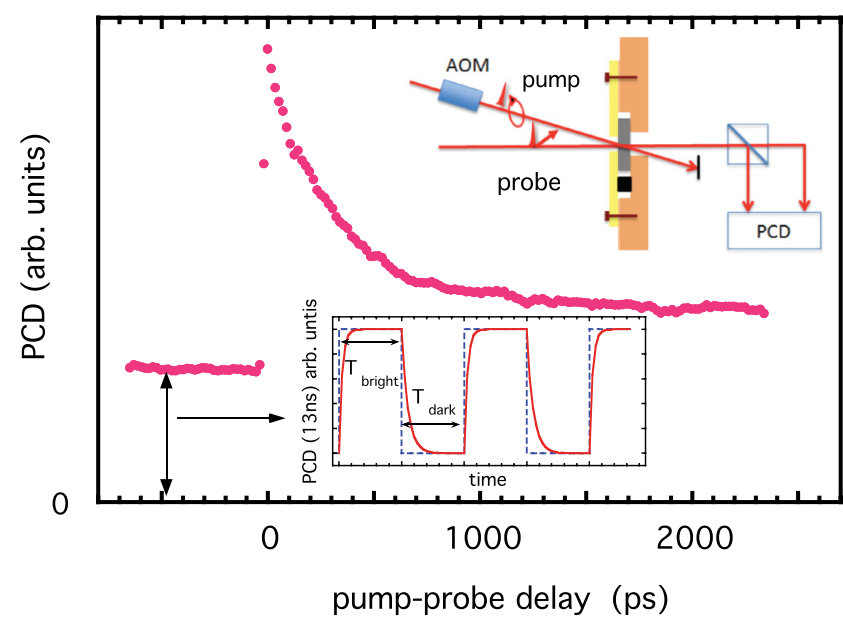

FIG. 1. (Color online) PCD signal vs pump-probe delay, obtained in sample $\mathrm{B}$, at $B=0 \mathrm{~T}$ and $2 \mathrm{~K}$, after a pulsed excitation at $1.32 \mathrm{eV}$. Insets: Scheme giving the principle of the PCD measurement and scheme of the temporal dependence of the model $\operatorname{PCD}\left(T_{L}\right)$ (solid line) imposed by the intensity modulation of the pump beam (dashed line).

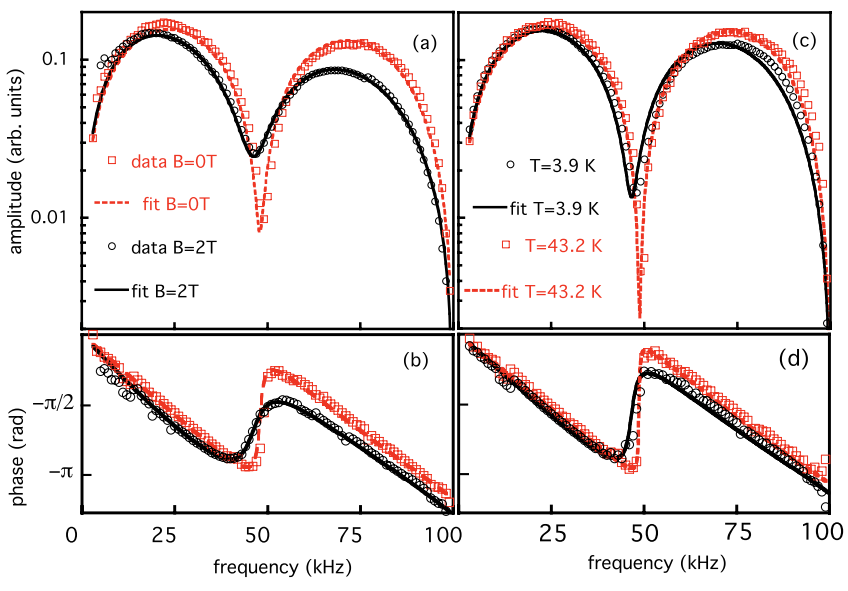

FIG. 2. (Color online) (a) and (b) (Sample B) Amplitude and phase of the second Fourier component of the experimental $\operatorname{PCD}\left(T_{L}\right)$ signal, at $2 \mathrm{~K}$, are shown as functions of modulation frequency $f_{m}$ for a fixed bright time of $10 \mu$ s and for two different magnetic fields ( $B=$ 0 and $2 \mathrm{~T}$ ). As discussed in Ref. 24, a second fitting parameter is the hole-spin initialization time $\tau_{i}$; it increases with magnetic field from 360 to 700 ns. (c) and (d) (Sample A) Amplitude and phase of the second Fourier component of the experimental $\operatorname{PCD}\left(T_{L}\right)$ signal are shown as functions of modulation frequency $f_{m}$ for a fixed bright time of $10 \mu \mathrm{s}$ and for two different temperatures ( 3.9 and $43.2 \mathrm{~K}$ ), at $B=0$. Here, $\tau_{i}$ decreases with temperature from 360 to $170 \mathrm{~ns}$. Experimental data shown in these figures have been obtained in similar conditions of excitation power.

function of the AOM modulation frequency $f_{m}$. As explained in Ref. 25, that is a good compromise between having a suitable signal-to-noise ratio and having enough characteristic features in the spectral function to assure a $T_{1}^{h}$-reliable determination. Finally, $T_{1}^{h}$ is obtained by fitting simultaneously the module and phase of the measured second Fourier component of the $\operatorname{PCD}\left(T_{L}\right)$ signal to the expected spectral function, when an exponential decay for the hole-spin polarization is assumed. This approach gives access to a characteristic relaxation time, but not to the full kinetics; nonetheless, the choice of an exponential decay, already observed, ${ }^{18}$ appears as well adapted to give the physical trends when an external parameter is applied. Moreover, for the fixed experimental conditions, when compared with a standard pump-probe configuration, the DTS technique gives very similar relaxation time while increasing the signal-to-noise ratio. ${ }^{25}$

In order to identify the mechanisms at the origin of the hole-spin relaxation, we have measured $T_{1}^{h}$ as a function of an external magnetic field along the growth direction (in the range of $0 \leqslant B \leqslant 2 \mathrm{~T})$, and also as a function of temperature $(4 \leqslant T$ $\leqslant 50 \mathrm{~K}$ ). Figures 2(a) and 2(b) show the measured and fitted second Fourier coefficient of the periodic $\operatorname{PCD}\left(T_{L}\right)$ signal, in absence of an applied magnetic field and for a magnetic field $B=2 \mathrm{~T}$, vs $f_{m}$. We note that, in the range 5 to $99 \mathrm{kHz}$, the amplitude of the second Fourier coefficient shows two maxima and a dip; meanwhile, the phase presents a discontinuity at the dip frequency $(\approx 50 \mathrm{kHz})$. Because the bright time is fixed at $10 \mu \mathrm{s}$, at $50 \mathrm{kHz}$, the pump modulation is a square-wave, with a $50 \%$ duty, and its second Fourier coefficient is zero. Then, in Fig. 2(a), a nonzero Fourier amplitude at $50 \mathrm{kHz}$ is the 

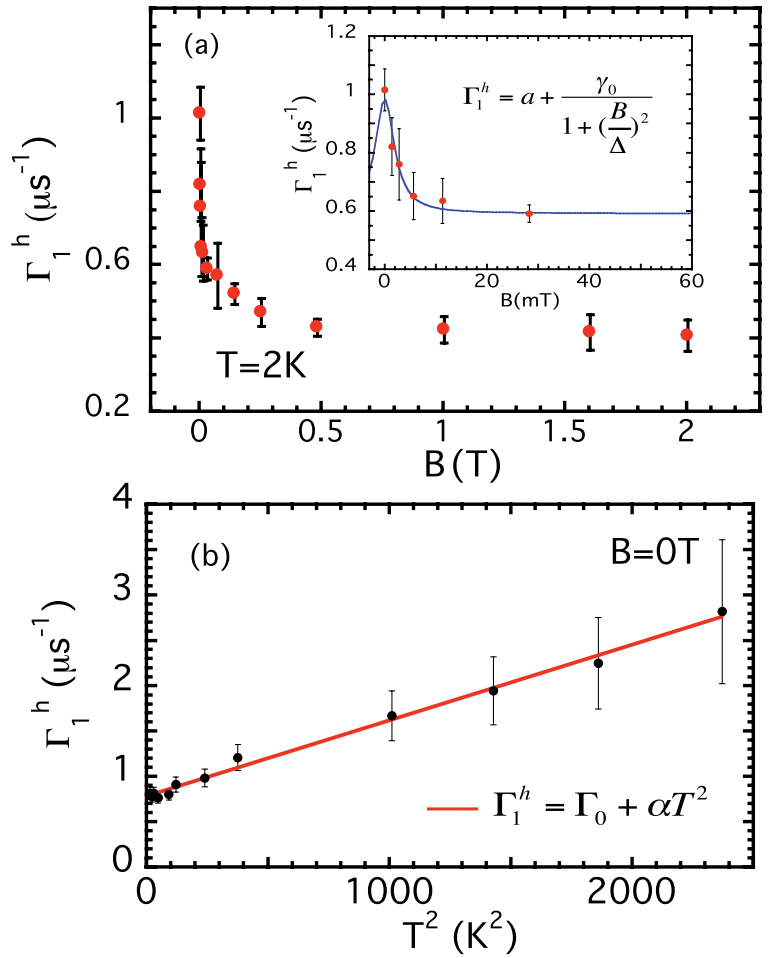

FIG. 3. (Color online) (a) (Sample B) $\Gamma_{1}^{h}=1 / T_{1}^{h}$ vs applied magnetic field, at $2 \mathrm{~K}$. Inset shows a magnification of the magneticfield dependence of $\Gamma_{1}^{h}$ from $B=0$ to $60 \mathrm{mT}$. Circles represent experimental data, and solid line the fit to the function given in the inset with $a=0.59 \mu \mathrm{s}^{-1}, \gamma_{0}=0.39 \mu \mathrm{s}^{-1}$ and $\Delta=2.3 \mathrm{mT}$. The error bars are determined experimentally. (b) (Sample A) $\Gamma_{1}^{h}$ as a function of square of the temperature, obtained in absence of an external magnetic field. The solid line shows a linear fit. The error bars are determined experimentally.

signature of a finite relaxation time $T_{1}^{h}$ in the $\operatorname{PCD}\left(T_{L}\right)$ signal. The greater the amplitude at $50 \mathrm{kHz}$, the longer the time $T_{1}^{h}$. Applying a 2-T magnetic field leads to an increase of the amplitude and a smoother discontinuity in the phase, both induced by a longer $T_{1}^{h} \cdot{ }^{25}$ Figure 3(a) shows $\Gamma_{1}^{h}=1 / T_{1}^{h}$ as a function of the applied magnetic field parallel to the growth direction. We clearly note three regimes. In the range from 0 to $10 \mathrm{mT}, \Gamma_{1}^{h}$ sharply decreases; between 10 and $500 \mathrm{mT}$, it slowly decreases, and at higher magnetic fields, $\Gamma_{1}^{h}$ remains constant at about $0.4 \mu \mathrm{s}^{-1}$. At $T=2 \mathrm{~K}$ and low magnetic field, the one-phonon process is negligible, as discussed previously. At $2 \mathrm{~K}$ and $0<$ $B<2$ T, the TPAM rate was predicted to be $\sim 10^{2}-10^{4} \mathrm{~s}^{-1},{ }^{26}$ and then TPAM cannot be responsible for the sharp decrease or the $0.4 \mu \mathrm{s}^{-1}$ plateau observed for $\Gamma_{1}^{h}$ in our samples.

We focus our discussion first on the sharp $\Gamma_{1}^{h}$ decrease in low longitudinal magnetic field. The inset of Fig. 3(a) shows a magnification of $\Gamma_{1}^{h}$ in the range of 0-60 mT. Experimental data are fitted by a Lorentzian function with half width at half maximum equal to $2.3 \mathrm{mT}$. This very small value of the magnetic field is comparable to the value needed to screen the transverse fluctuation of the $\mathrm{hf}$ interaction in this sample. ${ }^{5,6}$ Moreover, at zero magnetic field, the hf coupling acting at a longer time scale than the hole-spin dephasing time $T_{\Delta}^{h}$ (about $10 \mathrm{~ns}$ in the studied samples) has a characteristic time given by $T_{\Delta}^{h} \sqrt{N} \approx 2 \mu \mathrm{s}\left(N \approx 6.10^{4}\right.$ in our case $) .{ }^{3,4}$ At this timescale, at
$2 \mathrm{~K}$, we have already said that the TPAM is negligible; likewise, nuclear dipole-dipole interaction is responsible for nuclearbath dynamics in the ms scale. The measured relaxation rate $\Gamma^{h}$ is then associated with the dynamics of the nuclear-spin bath, controlled by hole-mediated nuclear-nuclear flip-flop, via second-order hyperfine coupling as calculated for an electron spin. ${ }^{28}$ Such flip-flop processes between two different nuclei modify the fluctuations of the nuclear-spin field only under the condition of inhomogeneity of the hole wave function. ${ }^{28}$ Because of this ascription, we would like now to come back to our assumption of an exponential decay for hole-spin polarization. In recent years, the spin polarization decay for an electron spin coupled with a nuclear bath has been the focus of numerous theoretical works, but despite this theoretical effort, the time shape of this decay remains an open question. Several laws have been proposed for the electron spin: power, inverse-log, or exponential; ${ }^{4,14,16}$ however, there is still no study concerning the hole spin. The decay law may also depend on the QD shape and the electron wave function, as discussed in Refs. 16 and 17. Then, taking into account the complex theoretical framework and the fact that our experimental data concern an ensemble of hole spins confined in InAs QDs, we have chosen an exponential decay law for the hole-spin polarization that should give the main trends of $T_{1}^{h}$ with variations of external parameters (magnetic field, temperature).

In Figure 3(a), we also observe a plateau: between $100 \mathrm{mT}$ and $2 \mathrm{~T}, \Gamma_{1}^{h}$ keeps almost constant at about $0.4 \mu \mathrm{s}^{-1}$. Secondorder hyperfine coupling between two nuclei is inversely proportional to the hole Zeeman splitting. ${ }^{28}$ At large magnetic field, a freezing of the nuclear-spin bath dynamics induced by this coupling is expected. Nonetheless, a finite value of the decay rate $\Gamma_{1}^{h}$ is observed when the applied magnetic field is large as compared to the fluctuating nuclear field. ${ }^{24}$ Such a behavior of the spin-relaxation time has been observed for an electron confined in InP QDs. ${ }^{18}$ There is no theoretical study of the magnetic-field behavior of $\Gamma_{1}^{h}$, imposed by hf interaction at the $\mu \mathrm{s}$ time scale; such a behavior is then difficult to interpret. As discussed previously, the spin-orbit mechanism is not at the origin of this plateau, but other processes associated with nearby impurities ${ }^{29}$ or with the electrostatic environment ${ }^{30}$ could be considered. Nevertheless, we have studied other samples, similar to sample B, with a distance between the QDs and $\delta$-doping planes equal to 2,4 , or $6 \mathrm{~nm}$ : no significant change in the field dependence of the decay rate $\Gamma_{1}^{h}$ has been observed. More studies are needed to explain the experimentally observed field dependence.

\section{B. Temperature dependence of the hole-spin relaxation rate}

To go further into the understanding of the hole-spin relaxation mechanisms, we have also studied the temperature dependence of $\Gamma_{1}^{h}$ in absence of an external magnetic field. Figures 2(c) and 2(d) show the measured and fitted spectral dependence of the second Fourier coefficient of the periodic $\operatorname{PCD}\left(T_{L}\right)$ signal, for two different temperatures 3.9 and $43.2 \mathrm{~K}$. We observe that the dip in amplitude and the discontinuity of the phase are more pronounced for higher temperature, indicating that $T_{1}^{h}$ is shorter at higher temperature. Figure 3(b) gives the temperature dependence of the measured $\Gamma_{1}^{h}$. In the 
range of $4-50 \mathrm{~K}, \Gamma_{1}^{h}$ increases with a quadratic dependence in temperature and can be fitted by $\Gamma_{1}^{h}(B=0)=\Gamma_{0}+\alpha T^{2}$ with $\Gamma_{0}=0.78 \mu \mathrm{s}^{-1}$ and $\alpha=8.4 \times 10^{-4} \mu \mathrm{s}^{-1} \mathrm{~K}^{-2}$. In QDs, for a weak magnetic field $B<1 \mathrm{~T}$ and for temperatures $T>2 \mathrm{~K}$, theoretical works predict that TPAM mediated by spin-orbit is the dominant hole-phonon interaction. ${ }^{21,26,31}$ The hole-spin in the initial state is promoted to a virtual intermediate state by absorption (emission) of a phonon; this intermediate state is also coupled with the final state by the emission (absorption) of another phonon. In contrast to the one-phonon process, even when the initial and final states are degenerate, virtual states participating in the second-order process allow the coupling between initial and final states. The hole-spin relaxation rate for TPAM is then proportional to the product of two Bose-Einstein factors for phonons, and then to the square of the temperature when $T \gg T_{\mathrm{ph}}$, with $T_{\mathrm{ph}}=\hbar c_{l} / k_{B} \lambda_{d}$ and $\lambda_{d}$, the hole-wave function radius. Assuming a parabolic confinement as in Ref. 25 and a typical energy separation $\hbar \omega_{0}=20 \mathrm{meV}$ between the first two confined hole states in the studied InAs QDs, we obtain $\lambda_{d}=4 \mathrm{~nm}$ (Ref. 32) and $T_{\mathrm{ph}} \approx 7 \mathrm{~K}$. The quadratic evolution is then expected from $T \geqslant 7 \mathrm{~K}$, as experimentally observed in Fig. 3(b). The extrapolation of the experimental values intercepts the vertical axis in Fig. 3(b) at a nonzero point. This zero-temperature relaxation rate is associated with hole-spin relaxation mediated by hyperfine coupling, as discussed previously, the TPAM being inefficient. As the temperature increases, the TPAM becomes dominant. Theoretical works ${ }^{25}$ have calculated the slope $\alpha$ of the linear fit in $T^{2}$, in the case $T \gg T_{\mathrm{ph}}$, it is written as $\alpha=\frac{0.3 \pi m_{h}^{6} \beta^{2} \Xi_{0}^{4} k_{B}^{2}}{\hbar^{6} c_{l}^{3} \lambda_{d} \rho_{c}^{2}}$ with $c_{l}$ the speed of sound in InAs, $\Xi_{0}$ the deformation potential, $\beta$ the spin-orbit coupling constant, $m_{h}$ the $\mathrm{HH}$ mass, and $\rho_{c}$ the InAs density. Hence, using parameters given in Ref. 26, we calculate $\alpha=(6.3 \pm 1.5) \times 10^{-4} \mu \mathrm{s}^{-1}$ $\mathrm{K}^{-2}$, in very good agreement with the experimental value. The TPAM for electrons is predicted to be much weaker than for holes and then negligible compared to hf effects. That is due to the weaker electron mass and its $\beta^{4}$ dependence ${ }^{33}$ instead of the already written $\beta^{2}$ dependence for a hole. Experimental signatures of these mechanisms for electron spins have been obtained in InP QDs. ${ }^{34}$

\section{CONCLUSION}

In summary, we have carried out a systematic study of the slow hole-spin relaxation in InAs QDs, in the regime of low magnetic field $(0 \leqslant B \leqslant 2 \mathrm{~T})$ and low temperature $(4 \leqslant T$ $\leqslant 50 \mathrm{~K}$ ). We have isolated two different contributions. The TPAM becomes the leading hole-spin relaxation mechanism at zero magnetic field and for temperatures higher than $7 \mathrm{~K}$, which is an experimental evidence of such a process for hole spin in QDs in very good agreement with recent theoretical predictions. The dynamics of the nuclear-spin bath, which is controlled by hole-mediated nuclear-nuclear flip-flop via second-order hf coupling, is at the origin of a relaxation rate $\Gamma_{1}^{h} \approx 1 \mu \mathrm{s}^{-1}$ and dominates at low temperature. The experimentally observed $\Gamma^{h}$ magnetic-field dependence, very similar to that observed by other authors for electron spin, should be the base for future theoretical works.

\section{ACKNOWLEDGMENTS}

We acknowledge M. Bernard for technical support in temperature measurement. This work is supported by the QUAMOS Project No. ANR-09-NANO-030 funded by the French National Agency (ANR).
*Corresponding author: maria.chamarro@insp.jussieu.fr

${ }^{1}$ Semiconductor Spintronics and Quantum Computation, edited by D. D. Awschalom, D. Loss, and N. Samarth (Springer, New York, 2002).

${ }^{2}$ M. Chamarro, F. Bernardot, and C. Testelin, J. Phys.: Condens. Matter 19, 445007 (2007).

${ }^{3}$ I. A. Merkulov, Al. L. Efros, and M. Rosen, Phys. Rev. B 65, 205309 (2002).

${ }^{4}$ A. V. Khaetskii, D. Loss, and L. Glazman, Phys. Rev. Lett. 88, 186802 (2002); Phys. Rev. B 67, 195329 (2003).

${ }^{5}$ B. Eble, C. Testelin, P. Desfonds, F. Bernardot, A. Balocchi, T. Amand, A. Miard, A. Lemaître, X. Marie, and M. Chamarro, Phys. Rev. Lett. 102, 146601 (2009).

${ }^{6}$ P. Desfonds, B. Eble, F. Fras, C. Testelin, F. Bernardot, M. Chamarro, B. Urbaszek, T. Amand, X. Marie, J. M. Gérard, V. Thierry-Mieg, A. Miard, and A. Lemaître, Appl. Phys. Lett. 96, 172108 (2010).

${ }^{7}$ E. A. Chekhovich, A. B. Krysa, M. S. Skolnick, and A. I. Tartakovskii, Phys. Rev. Lett. 106, 027402 (2011).

${ }^{8}$ P. Fallahi, S. T. Yilmaz, and A. Imamoglu, Phys. Rev. Lett. 105, 257402 (2010).
${ }^{9}$ J. Fischer, W. A. Coish, D. V. Bulaev, and D. Loss, Phys. Rev. B 78, 155329 (2008).

${ }^{10}$ C. Testelin, F. Bernardot, B. Eble, and M. Chamarro, Phys. Rev. B 79, 195440 (2009), and references therein concerning HH-LH mixing.

${ }^{11}$ T. Belhadj, T. Amand, A. Kunold, C.-M. Simon, T. Kuroda, M. Abbarchi, T. Mano, K. Sakoda, S. Kunz, X. Marie, and B. Urbaszek, Appl. Phys. Lett. 97, 051111 (2010).

${ }^{12}$ The mentioned calculations concern only the hf electron spin interaction. Up to now there is no equivalent calculation for the hole spin. Nevertheless, for the particular case of a mixed HH-LH state, having the main trends seem probable.

${ }^{13}$ S. I. Erlingsson and Y. V. Nazarov, Phys. Rev. B 70, 205327 (2004).

${ }^{14}$ W. A. Coish and D. Loss, Phys. Rev. B 70, 195340 (2004).

${ }^{15}$ C. Deng and X. Hu, Phys. Rev. B 73, 241303 (2006).

${ }^{16}$ K. A. Al-Hassanieh, V. V. Dobrovitski, E. Dagotto, and B. N. Harmon, Phys. Rev. Lett. 97, 037204 (2006).

${ }^{17}$ G. Chen, D. L. Bergman, and L. Balents, Phys. Rev. B 76, 045312 (2007).

${ }^{18}$ B. Pal, S. Yu. Verbin, I. V. Ignatiev, M. Ikezawa, and Y. Masumoto, Phys. Rev. B 75, 125322 (2007). 
${ }^{19}$ M. Kroutvar, Y. Ducommun, D. Heiss, M. Bichler, D. Schuh, G. Abstreiter, and J. J. Finley, Nature 432, 81 (2004).

${ }^{20}$ D. Heiss, S. Schaeck, H. Huebl, M. Bichler, G. Abstreiter, J. J. Finley, D. V. Bulaev, and D. Loss, Phys. Rev. B 76, 241306(R) (2007).

${ }^{21}$ A. V. Khaetskii and Y. V. Nazarov, Phys. Rev. B 64, 125316 (2001).

${ }^{22}$ L. M. Woods, T. L. Reinecke, and Y. Lyanda-Geller, Phys. Rev. B 66, 161318 (2002).

${ }^{23}$ D. V. Bulaev and D. Loss, Phys. Rev. Lett. 95, 076805 (2005).

${ }^{24}$ On completion of this manuscript, we became aware of hole-spin related experiments: Y. Li, N. Sinitsyn, D. L. Smith, D. Reuter, A. D. Wieck, D. R. Yakovlev, M. Bayer, S. A. Crooker, Phys. Rev. Lett. 108, 186603 (2012).

${ }^{25}$ F. Fras, B. Eble, P. Desfonds, F. Bernardot, C. Testelin, M. Chamarro, A. Miard, and A. Lemaître, Phys. Rev. B 84, 125431 (2011).

${ }^{26}$ M. Trif, P. Simon, and D. Loss, Phys. Rev. Lett. 103, 106601 (2009).

${ }^{27}$ B. Eble, P. Desfonds, F. Fras, F. Bernardot, C. Testelin, M. Chamarro, A. Miard, and A. Lemaître, Phys. Rev. B 81, 045322 (2010).
${ }^{28}$ R.-B. Liu, W. Yao, and L. J. Sham, New J. of Phys. 9, 226 (2007).

${ }^{29}$ H. S. Nguyen, G. Sallen, C. Voisin, P. Roussignol, C. Diederichs, and G. Cassabois, Phys. Rev. Lett. 108, 057401 (2012).

${ }^{30}$ J. Houel, A. V. Kuhlmann, L. Greuter, F. Xue, M. Poggio, B. D. Gerardot, P. A. Dalgarno, A. Badolato, P. M. Petroff, A. Ludwig, D. Reuter, A. D. Wieck, and R. J. Warburton, Phys. Rev. Lett. 108, 107401 (2012)

${ }^{31}$ H. Wei, M. Gong, G.-C. Guo, and L. He, Phys. Rev. B 85, 045317 (2012).

${ }^{32}$ For a Gaussian wavefunction [H. Y. Ramirez, C. H. Lin, C. C. Chao, Y. Hsu, W. T. You, S. Y. Huang, Y. T. Chen, H. C. Tseng, W. H. Chang, S. D. Lin, and S. J. Cheng, Phys. Rev. B 81, 245324 (2010)], as for a parabolic potential in Ref. 25, it has been shown that the radius $\lambda_{d}$ is typically one quarter of the crystallographic QD diameter $d$. Then, $\lambda_{d}=4 \mathrm{~nm}$ corresponds to $d \approx 15 \mathrm{~nm}$.

${ }^{33}$ P. San-José, G. Zarand, A. Shnirman, and G. Schön, Phys. Rev. Lett. 97, 076803 (2006).

${ }^{34}$ B. Pal, M. Ikezawa, Y. Masumoto, and I. V. Ignatiev, J. Phys. Soc. Jpn. 75, 054702 (2006) 